\title{
The Hidden Costs of High Fructose Corn Syrup: Challenges to Energy Balance and Fat Mobilization from Adipose Tissue
}

Robert A Orlando* and William S Garver

Department of Biochemistry and Molecular Biology, School of Medicine, University of New Mexico, MSC08 4670, 1 Albuquerque, New Mexico, 87131, USA

The most recent report from the Centers for Disease Control and Prevention [1], based on National Health and Nutrition Examination Surveys (NHANES), shows that over 78 million U.S. adults now meet the criteria as clinically obese, more than double the number from 20 years ago. Arguably even more daunting is the fact that over 12 million of our children, ages 2-19, are now classified as obese. Clearly we have surpassed the epidemic stage [2] and have now entered a serious multigenerational problem. Human obesity is a complex problem involving physiological, psychological and behavioral contributions [3]. Because of this complexity, many proposals have been presented as to how we arrived at this state. However, if we reduce the complexity of this human condition in terms of energy balance, our obesity dilemma can be summed by the first law of thermodynamics: for a system to remain in equilibrium, the amount of energy added to a system must equal the sum of the amount of energy lost due to work by the system (physical activity) plus the internal energy expenditure of the system (basal metabolic rate). Thus, a negative energy balance will draw from energy stores resulting in weight loss, whereas a positive energy balance will result in weight gain as the body stores unused energy for later periods of negative energy balance [4]. From this fundamental principle, it is not hard to see how over consumption of calorie-dense foods and a shift to a more sedentary lifestyle are fueling our obesity problem.

Early in human evolution, our dietary needs were met from hunting/ gathering activities, which were subject to varying levels of energy output demands and seasonal influences [5]. The calories provided by hunting/gathering were sufficient to fulfill our immediate energy requirements and possibly a bit more for energy storage permitting longer times between meals. The need for energy storage became more critical as populations adopted migratory behaviors to seek new territories for growth and as agrarian cultures began to take foothold and became subject to climate variations from year to year. Climate variations are often referred to as "feast or famine" periods; when water and temperatures are optimal, agrarian activities flourished, however, when these same populations encountered drought and/or disease, food sources became sorely limited. The durations of these seasonal variations were unpredictable and survival of the population was dependent on inheritance of metabolic traits that provided advantages to weather the periods of limited food availability. These genetic traits favored individuals who could efficiently store excess calories consumed during times of plenty [6].

The culmination of many years of metabolic research has provided an elegant and detailed map defining how consumed calories are processed. The metabolic handling of dietary calories depends on the molecular form of the calories ingested, e.g. carbohydrates or fats, our two primary energy sources. Carbohydrates represent our energy choice for the immediate and rapid production of ATP. When the intracellular energy charge is sufficiently elevated, ATP production slows and the energy capacity of carbohydrates is stored as glycogen for later use when energy demands require recharging the ATP pool. Because the carbohydrate storage capacity in the body is limited (primarily restricted to liver and skeletal muscle), once glycogen pools are filled, excess carbohydrates are metabolized by hepatocytes and converted to fatty acids for long-term storage in adipose depots. Dietary fats can also serve as an immediate energy source for some tissues, but the majorities are delivered to adipose tissue for storage, ready to fuel longterm fasting needs. Satisfying our dietary needs for energy production and storage are powerful, directive forces in the evolutionary process and when in balance work seamlessly to provide what is needed for the body to thrive. However, when this metabolic balance is lost due to chronic over- or under-consumption, various disease processes begin. While under-consumption is rarely a problem in Western cultures, caloric over-consumption feeds into our natural survival mechanisms leading to excess storage of energy-dense molecules and, left unchecked, ultimately to an obese condition.

Over consumption is often mistakenly attributed to simply ingesting large volumes of foodstuffs, such as our traditional holiday meals. More correctly, over consumption is best defined as caloric intake over and above what is needed to fulfill our short- and longterm metabolic demands. Foods that have been processed to include added carbohydrates and fats in excess of what is found in their natural, unprocessed state can greatly contribute to over-consumption of calories. These calorie-dense foods typically have longer shelf lives supporting a strong commercial interest and provide both mental and physical satisfaction, giving them a sense of being "comfort foods" [7]. One example of food processing that has come under intense scrutiny and subject to vigorous debate between researchers, clinicians and commercial producers is the inclusion of High Fructose Corn Syrup (HFCS) as a sweetener in foods and beverages [8,9]. Some proponents argue that HFCS is a natural sugar as it contains the same carbohydrate composition as common table sugar, sucrose (disaccharide of glucose and fructose as $\alpha$-D-glucopyranosyl-(1,2)- $\beta$-D-fructofuranoside). By contrast, opponents claim that HFCS is an unnatural source of carbohydrates and its inclusion in our diet is the major cause of hyperglycemia and uncontrolled weight gain. Perhaps the best approach to address this argument begins with re-examining how HFCS is made.

HFCS was first introduced by Marshall and Kooi [10], although commercial production did not commence until the 1970's when Dr. Yoshiyuki Takasaki successfully scaled the production process. HFCS is produced by first milling corn to generate corn starch, which is then enzymatically digested using a-amylase and glucoamylase to produce corn syrup, a solution consisting almost entirely of glucose.

*Corresponding author: Robert A Orlando, Department of Biochemistry and Molecular Biology, School of Medicine, University of New Mexico, MSC08 4670, 1 Albuquerque, New Mexico, 87131, USA, Tel: 505-272-5593, Fax: 505-272-5593. E-mail: rorlando@salud.unm.edu

Received April 03, 2013; Accepted April 23, 2013; Published April 28, 2013

Citation: Orlando RA, Garver WS (2013) The Hidden Costs of High Fructose Corn Syrup: Challenges to Energy Balance and Fat Mobilization from Adipose Tissue. J Biomol Res Ther 1: e110. doi:10.4172/2167-7956.1000e110

Copyright: (c) 2013 Orlando RA, et al. This is an open-access article distributed under the terms of the Creative Commons Attribution License, which permits unrestricted use, distribution, and reproduction in any medium, provided the original author and source are credited. 
The glucose in corn syrup is next isomerized into varying percentages of fructose by controlled enzymatic treatment with xylose isomerase to produce HFCS. The ratios of glucose and fructose in the HFCS are then adjusted to formulate the desired product. For soft drinks, HFCS 55 is used as a sweetener consisting of $55 \%$ fructose, $42 \%$ glucose. HFCS 42 ( $42 \%$ fructose, $55 \%$ glucose) is typically used for processed foods, cereals and baked goods. For some processed foods, higher concentrations of fructose are used to intensify the sweetness. Fructose is a popular commercial choice because it is 1.73 times sweeter by taste than is sucrose [11] making fructose more palatable and satisfying for sweetness cravings [12].

Once fructose is absorbed by intestinal enterocytes and enters the portal circulation, it is rapidly taken up by the liver with little passing into the systemic circulation [13], making the liver the major site for metabolizing dietary fructose. In humans, the metabolic handling of fructose by hepatocytes is slightly different than that of glucose, yet the end products are similar. Upon entering hepatocytes through the glucose transporter-2 (GLUT-2; in contrast to the GLUT-5 fructose transporter in intestinal epithelia), fructose is trapped within the cell through phosphorylation by fructokinase becoming fructose-1-phosphate (Figure 1). Unlike glucose-6-phosphate, fructose-1-phosphate bypasses phosphofructokinase activity of glycolysis and is metabolized directly into two trioses (glyceraldehyde and dihydroxyacetone phosphate) by fructose-1-phosphate aldolase. It has been reported that these two trioses can enter gluconeogenesis for synthesis of a small amount of glucose [14] and contribute to hepatic glycogen stores [15]; however, little of this fructose-derived glucose enters the general circulation [16]. Alternatively, and more likely during the high insulin, post-prandial state, the two trioses are used for de novo fatty acid synthesis [17] and their energy capacity stored long-term as triacylglycerol in adipose tissue [18]. In either case, whether converted to glucose for glycogen storage or its carbons used for fat synthesis, fructose follows normal physiologic pathways for energy utilization or storage. If this is the case, then why is ingestion of HFCS so strongly associated with obesity and hyperlipidemia? Consider first the quantities of HFCS ingested and its metabolic fate. Circulating glucose is cleared from the circulation within



Figure 1: Comparative metabolic processing of glucose and fructose by hepatocytes. Following GLUT-2 mediated transport into hepatocytes and phosphorylation by their respective kinases, the metabolic pathways of glucose and fructose diverge. In response to high intracellular energy charge, glucose catabolism in glycolysisis reduced due to regulation of phosphofructokinase, making glucose fully available for glycogen synthesis. By contrast, the majority of fructose bypasses this key regulatory step and is metabolized to synthesize fatty acids (large arrows). Only a small amount of fructose contributes to glycogen stores (dashed lines) and largely during a fasting state when gluconeogenesis is active.
2-3 hours following a meal under the influence of insulin signaling. Insulin responsive tissues, primarily muscle and adipose, translocate GLUT-4 transporters to their surfaces to capture and assimilate glucose into glycogen or triacylglycerides, respectively. By contrast, uptake of fructose is not insulin sensitive thereby excluding muscle, the largest quantitative storage site for carbohydrates, from removing fructose from the circulation. This being the case, the metabolic fate of fructose is left to the liver of which the majority is converted into fatty acids for packaging into VLDL particles and extra hepatic transport. Thus, from the consequences of fructose metabolism we can redefine caloriedense additives, such as HFCS, as not only supplying extra calories by virtue of extra dietary caloric load, but also as supplying extra energy that is not efficiently burned by the major energy-consuming organs and ends up largely as stored fat. Indeed, clinical evidence confirms that consumption of HFCS is directly associated with significantly elevated fasting lipid levels and greater adiposity [19].

So, if fructose metabolism leads to excessive de novo fat synthesis and storage, can we solve the growing adiposity problem in our culture by simply increasing the body's burn rate through physical exertion while still satisfying our craving for the sweetness provided by HFCS? This may appear as a simple, logical and manageable solution, but aside from the difficulties in sustaining dietary modifications, there may be yet another hidden pathologic consequence of fructose metabolism that augments the difficulties in losing excess adipose through exercise. Conventional wisdom argues that exercise reduces adiposity by increasing metabolic activity and in turn the burn rate of stored calories. By depleting intracellular energy stores, glycogen is utilized for immediate ATP production and fat is mobilized from adipose depots following endocrine stimulation. Mobilized fatty acids are taken up and oxidized to supplement glucose during energy production. Thus, according to the first law of thermodynamics, if an individual increases their energy utilization through increased activity, then stored energy (fat) will be consumed as needed to balance the energy equation. However, studies investigating lipid mobilization from adipose depots show that fat release does not follow simple rules such as all fatty acids are equal when it comes to mobilization from storage depots. Rather, mobilization rates of fatty acids upon endocrine stimulation are highly dependent on their molecular structure [20-22] as opposed to the historical concept of "first in, first out" [23]. The general rule that has come about from these studies is that mobilization decreases with increasing chain length for a given degree of unsaturation and increases with increasing unsaturation for a given chain length $[24,25]$. The data argues that mobilization is highly dependent on the physicochemical properties of the individual fatty acid structure and that release from adipose stores is largely influenced by thermodynamic constraints rather than fatty acid availability or accessibility within the store With these basic tenets of fatty acid properties, the data reported on selective fatty acid mobilization presents an interesting conundrum for fatty acids derived from fructose metabolism. While dietary fats contain a variety of chain lengths and degrees of unsaturation (mono- and polyunsaturated structures), de novo fatty acid synthesis by hepatocytes is limited with regard to fatty acid structure, these being largely restricted to 16- and 18-carbon species. In addition, desaturase activity introduces only limited number of double bonds. Thus, newly synthesized fatty acids, whether from fructose or glucose, are categorized as medium-long chain length with a relatively high degree of saturation. According to the evidence, fatty acids with these physicochemical properties are mobilized to a lesser degree than fatty acids derived from dietary sources, especially when compared with those from the polyunsaturated class [25]. In the context of fructose 
Citation: Orlando RA, Garver WS (2013) The Hidden Costs of High Fructose Corn Syrup: Challenges to Energy Balance and Fat Mobilization from Adipose Tissue. J Biomol Res Ther 1: e110. doi:10.4172/2167-7956.1000e110

derived fatty acids, selective mobilization then means that other fatty acids will be preferentially released for energy needs over those synthesized endogenously using fructose as the carbon source. In this case, mobilization of medium-long chain, mostly saturated fatty acids will present a significant thermodynamic challenge even when stimulated by fasting and/or exercise. These thermodynamic challenges present serious difficulties to those who wish to reduce their adiposity while still consuming HFCS-containing foods. One can imagine that over many years of HFCS consumption, an individual's adipose depots are likely to contain greater percentages of fructose-derive fatty acids and thus exacerbate the challenges of mobilization. Add to these challenges age-dependent decreases in both basal metabolic rates [26] and overall ability to mobilize fatty acids during beta-adrenergic stimulation [27], and the scales of energy balance tip further into promoting obesity.

Measurements of thermodynamic barriers to fatty acid mobilization have not been determined as yet, but represent an important parameter for a better understanding of the consequences of HFCS and excess carbohydrate-derived caloric intake in general. Additional studies on intracellular lipase activities, including adipose triglyceride lipase and hormone-sensitive lipase, should provide greater details as to how these enzymes function at the lipid-cytosolic interface and provide clues as to how their activities might be therapeutically enhanced to overcome these thermodynamic barriers. And finally, one immediate way we can begin to address this problem is by increasing dietary consumption of "good" fats, those derived from plant sources with high degrees of unsaturation [28]. Evidence indicates that the fatty acid composition of human adipose depots generally reflects dietary intake patterns [2931], and thus, if a higher percentage of polyunsaturated fats is included in the diet, along with a reduction in HFCS, we might expect to reverse adipose fat composition into one with more favorable physicochemical properties.

\section{Acknowledgements}

The authors declare no competing financial interests.

\section{References}

1. Ogden CL, Carroll MD, Kit BK, Flegal KM (2012) Prevalence of Obesity in the United States, 2009-2010. U.S. Department of Health and Human Services, Centers for Disease Control and Prevention, National Center for Health Statistics, USA.

2. Mitchell NS, Catenacci VA, Wyatt HR, Hill JO (2011) Obesity: overview of an epidemic. Psychiatr Clin North Am 34: 717-732.

3. Boulos R, Vikre EK, Oppenheimer S, Chang H, Kanarek RB (2012) ObesiTV: how television is influencing the obesity epidemic. Physiol Behav 107: 146-153.

4. Hill JO, Wyatt HR, Peters JC (2012) Energy balance and obesity. Circulation 126: 126-132.

5. Heitmann BL, Westerterp KR, Loos RJ, Sorensen TI, O'Dea K, et al. (2012) Obesity: lessons from evolution and the environment. Obes Rev 13: 910-922.

6. Speakman JR, O'Rahilly S (2012) Fat: an evolving issue. Dis Model Mech 5: 569-573.

7. Gibson EL (2012) The psychobiology of comfort eating: implications for neuropharmacological interventions. Behav Pharmacol 23: 442-460.

8. Brice M (2012) Sweet Debate Rages On: Scientists Say High-Fructose Corn Syrup Not to Blame for Obesity Epidemic.
9. Rippe JM, Angelopoulos TJ (2013) Sucrose, high-fructose corn syrup, and fructose, their metabolism and potential health effects: what do we really know? Adv Nutr 4: 236-245.

10. Marshall RO, Kooi ER (1957) Enzymatic conversion of D-glucose to D-fructose. Science 125: 648-649.

11. Hanover LM, White JS (1993) Manufacturing, composition, and applications of fructose. Am J Clin Nutr 58: 724S-732S

12. Lustig RH (2010) Fructose: metabolic, hedonic, and societal parallels with ethanol. J Am Diet Assoc 110: 1307-1321.

13. Tappy L, Le KA (2010) Metabolic effects of fructose and the worldwide increase in obesity. Physiol Rev 90: 23-46.

14. Delarue J, Normand S, Pachiaudi C, Beylot M, Lamisse F, et al. (1993) The contribution of naturally labelled $13 \mathrm{C}$ fructose to glucose appearance in humans. Diabetologia 36: 338-345.

15. Coss-Bu JA, Sunehag AL, Haymond MW (2009) Contribution of galactose and fructose to glucose homeostasis. Metabolism 58: 1050-1058.

16. Tounian P, Schneiter P, Henry S, Jequier E, Tappy L (1994) Effects of infused fructose on endogenous glucose production, gluconeogenesis, and glycogen metabolism. Am J Physiol 267: E710-E717.

17. Parks EJ, Skokan LE, Timlin MT, Dingfelder CS (2008) Dietary sugars stimulate fatty acid synthesis in adults. J Nutr 138: 1039-1046.

18. Bremer AA, Mietus-Snyder M, Lustig RH (2012) Toward a unifying hypothesis of metabolic syndrome. Pediatrics 129: 557-570.

19. Stanhope KL, Schwarz JM, Keim NL, Griffen SC, Bremer AA, et al. (2009) Consuming fructose-sweetened, not glucose-sweetened, beverages increases visceral adiposity and lipids and decreases insulin sensitivity in overweight/ obese humans. J Clin Invest 119: 1322-1334.

20. Raclot T, Groscolas R (1995) Selective mobilization of adipose tissue fatty acids during energy depletion in the rat. J Lipid Res 36: 2164-2173.

21. Raclot T, Langin D, Lafontan M, Groscolas R (1997) Selective release of human adipocyte fatty acids according to molecular structure. Biochem J 324: 911-915

22. Raclot T, Mioskowski E, Bach AC, Groscolas R (1995) Selectivity of fatty acid mobilization: a general metabolic feature of adipose tissue. Am J Physiol 269 : R1060-R1067.

23. Ekstedt B, Olivecrona T (1970) Uptake and release of fatty acids by rat adipose tissue: last in to first out? Lipids 5: 858-860.

24. Lafontan M, Langin D (2009) Lipolysis and lipid mobilization in human adipose tissue.Prog Lipid Res 48: 275-297.

25. Raclot T (2003) Selective mobilization of fatty acids from adipose tissue triacylglycerols.Prog Lipid Res 42: 257-288.

26. Fry CS, Rasmussen BB (2011) Skeletal muscle protein balance and metabolism in the elderly. Curr Aging Sci 4: 260-268.

27. Blaak EE (2000) Adrenergically stimulated fat utilization and ageing. Ann Med 32: $380-382$

28. Buckley JD, Howe PR (2009) Anti-obesity effects of long-chain omega-3 polyunsaturated fatty acids. Obes Rev 10: 648-659.

29. Fernandez-Quintela A, Churruca I, Portillo MP (2007) The role of dietary fat in adipose tissue metabolism. Public Health Nutr 10: 1126-1131.

30. Field CJ, Goruk SD, Wierzbicki AA, Clandinin MT (1989) The effect of dietary fat content and composition on adipocyte lipids in normal and diabetic states. Int J Obes 13: 747-756.

31. Hodson L, Skeaff CM, Fielding BA (2008) Fatty acid composition of adipose tissue and blood in humans and its use as a biomarker of dietary intake. Prog Lipid Res 47: 348-380. 